\title{
A Note on Inflation with Tachyon Rolling on the Gauss-Bonnet Brane
}

\author{
B.C. Paul \\ Physics Department, North Bengal University, Dist. : Darjeeling, Pin : 734 430, INDIA * \\ M.Sami \\ Post Bag 4, Ganeshkhind, \\ Pune $41100 \%$, India
}

\begin{abstract}
In this paper we study the tachyonic inflation in brane world cosmology with Gauss-Bonnet term in the bulk. We obtain the exact solution of slow roll equations in case of exponential potential. We attempt to implement the proposal of Lidsey and Nunes[1] for the tachyon condensate rolling on the Gauss-Bonnet brane and discuss the difficulties associated with the proposal.

PACS numbers: 98.80.Cq, 98.80.Hw, 04.50.+h
\end{abstract}

\section{INTRODUCTION}

At present there seems to be no viable alternative to inflationary scenario. But inspite of all the attractive features of cosmological inflation, its mechanism of realization still remains to be ad hoc. As inflation operates at Planck's scale, the needle of hope points towards the string theory. It is, therefore, not surprising that $\mathrm{M} /$ String theory inspired models are under active consideration in cosmology at present. It was recently been suggested that rolling tachyon condensate, in a class of string theories, may have interesting cosmological consequences [2]. Rolling tachyon matter associated with unstable D-branes has an interesting equation of state which smoothly interpolates between -1 and 0 . The tachyonic matter, therefore, might provide an explanation for inflation at the early epochs and could contribute to some new form of cosmological dark matter at late time 3 . Unfortunately, this scenario faces difficulties associated with generation of enough inflation, reheating and the formation of caustics/kinks.

Another interesting development in cosmology inspired by String theory is related to Brane World cosmology. In this picture all the matter fields are confined to the brane whereas gravity can propagate in the bulk. The scenario has interesting cosmological implications, in particular, the prospects of inflation are enhanced on the brane due to the modifications in the Friedmann equation. While discussing the applications of brane-worlds, one often assumes Einstein Gravity in the bulk and then projects the dynamics on to the brane. This leads to the high energy corrections in the Friedman equation which changes the expansion dynamics in the early universe. To be in the better spirit with string theory, one should include

\footnotetext{
*Electronic address: bcpaul@iucaa.ernet.in

${ }^{\dagger}$ On leave from: Department of Physics, Jamia Millia, New Delhi110025; Electronic address: sami@iucaa.ernet.in
}

the higher order curvature invariants to the EinsteinHilbert action [5]. The Gauss-Bonnet gravity projected on the brane leads to modified Friedmann equation different from its counter part in the RS scenario [6]. And this may have interesting cosmological consequences. As recently demonstrated by Lidsey and Nunes [1], the GaussBonnet modified expansion dynamics can lead to spectral index of perturbation spectrum consistent with the recent WMAP observation. Interestingly, this is achieved by suitably fixing the Gauss-Bonnet coupling parameter and the brane tension without tuning the slope of the scalar field potential that drives inflation. As mentioned above, there are problems with tachyonic inflation as there is no free parameter in the tachyonic potential to tune to make the field roll slow allowing the required number of inflationary e-foldings. The proposal of Lidsey and Nunes [1] is specially interesting in case of tachyonic inflation as it does not require the tuning of the slope of potential. In this note we study the tachyonic inflation with exponential potential on Gauss-Bonnet brane and show that the spectral index of scalar density perturbations can be pushed close to unity. However, for the physical relevant values of parameters, one requires to tune the slope of the potential.

\section{A. Brane World with Gauss-Bonnet Term in the Bulk}

We consider five dimensional bulk action with GaussBonnet term given by

$$
\begin{aligned}
S_{M} & =\frac{1}{2 \kappa_{5}^{2}} \int_{M} d^{5} x \sqrt{-g}\left[R-2 \Lambda+\alpha\left(R^{2}-4 R_{\alpha \beta} R^{\alpha \beta}\right.\right. \\
& \left.\left.+R_{\alpha \beta \gamma \delta} R^{\alpha \beta \gamma \delta}\right)\right]+S_{\partial M}+S_{\text {matter }}
\end{aligned}
$$

where $\alpha$ represents the Gauss-Bonnet coupling, $\Lambda$ is the bulk cosmological constant. The additive pieces of the action $S_{\partial M}$ and $S_{\text {matter }}$ represent the action on the boundary and the matter part respectively. The effective Fried- 
mann equation is obtained by imposing a $\mathbf{Z}_{2}$ symmetry across the brane $[1,6]$

$$
H^{2}=\frac{c_{+}+c_{-}-2}{8 \alpha}
$$

where

$$
c_{ \pm}=\left(\left[\left(1+\frac{4}{3} \alpha \Lambda\right)^{3 / 2}+\frac{\alpha}{2} \kappa_{5}^{4} \sigma^{2}\right]^{1 / 2} \pm \sqrt{\frac{\alpha}{2}} \kappa_{5}^{2} \sigma\right)^{2 / 3}
$$

and $\sigma$ represents the energy density of the matter sources.

The conservation of energy-momentum on the brane for perfect fluid matter sources is given by

$$
\dot{\sigma}+3 H(\sigma+p)=0
$$

where $p$ represents the pressure of the fluid and $\sigma$ its energy density.

The cosmic dynamics on the brane is fully determined using equations (2) and (4) when the equation of state is known. We use here a simplified approach following Lidsey and Nunes 1]. Accordingly we define a new variable and write the energy density as

$$
\sigma=\sqrt{\left(\frac{2 b}{\alpha \kappa_{5}^{4}}\right)} \sinh x, \quad b=\left(1+\frac{4}{3} \alpha \Lambda\right)^{3 / 2}
$$

Consequently the Friedmann equation (2) takes a simple form which is given by

$$
H^{2}=\frac{1}{4 \alpha}\left[b^{\frac{1}{3}} \cosh \left(\frac{2 x}{3}\right)-1\right] .
$$

Taking into account (5) and retaining the terms up to second order in $\sigma$ in equation (6) we have 1 ]

$$
H^{2}=\frac{\kappa_{4}^{2}}{3} \rho\left[1+\frac{\rho}{2 \lambda}\right]+\frac{\Lambda_{4}}{3},
$$

where $\sigma$ is assumed to be decomposed into matter contribution $\rho$ and the brane tension $\lambda, \sigma=\lambda+\rho$ and the four-dimensional cosmological constant is given by

$$
\Lambda_{4}=\frac{3}{4 \alpha}\left(b^{\frac{1}{3}}-1\right)+\frac{\kappa_{5}^{4}}{12 b^{2 / 3}} \lambda^{2} .
$$

The above equation reduces to the standard form of the Friedmann equation at a sufficiently low energy scales $(\rho<<\lambda)$ with

$$
\kappa_{4}^{2}=\frac{1}{M_{P}^{2}}=\frac{\kappa_{5}^{4} \lambda}{6 b^{\frac{2}{3}}}
$$

where $M_{P}$ is the four-dimensional reduced Planck mass. It is also found that the four dimensional cosmological constant vanishes when the brane tension satisfies

$$
\lambda=\frac{3}{2 \alpha \kappa_{4}^{2}}\left[1-b^{\frac{1}{3}}\right] .
$$

\section{TACHYONIC INFLATION}

The energy density $\rho$ and pressure p for tachyonic field are given by

$$
\rho=\frac{V(\phi)}{\sqrt{1-\dot{\phi}^{2}}}, \quad p=-V(\phi) \sqrt{1-\dot{\phi}^{2}}
$$

The field evolution equation (equivalent to equation (4) is

$$
\frac{\ddot{\phi}}{1-\dot{\phi}^{2}}+3 H \dot{\phi}+\frac{V_{, \phi}}{V(\phi)}=0
$$

We now describe inflation on the brane assuming slow roll approximation. Using equations (5) and (9) we obtain

$$
V=V_{0} \sinh x \equiv \sqrt{\left(\frac{\lambda b^{1 / 3}}{3 \alpha \kappa_{4}^{2}}\right)} \sinh x
$$

The slow-roll parameters in this case become(we here use the same definition of slow roll as in Ref 1 ].

$$
\epsilon=\left(\frac{2 \lambda}{\kappa_{4}^{2}} \frac{V_{, \phi}^{2}}{V^{4}}\right) \epsilon_{G B}, \quad \eta=\left(\frac{2 \lambda}{\kappa_{4}^{2}}(\ln V), \phi \phi\right) \eta_{G B}
$$

where $\epsilon_{G B}$ and $\eta_{G B}$ are given by

$$
\begin{gathered}
\epsilon_{G B}=\left[\frac{2 b^{2 / 3}}{27} \frac{\sinh (2 x / 3) \tanh x \sinh ^{2} x}{\left(b^{1 / 3} \cosh (2 x / 3)-1\right)^{2}}\right] \\
\eta_{G B}=\left[\frac{4 \alpha}{3\left(b^{1 / 3} \cosh (2 x / 3)-1\right)}\right]
\end{gathered}
$$

The number of e-folds of inflationary expansion, is obtained using equations (6) and (12), which is given by

$$
N(x)=-\frac{3}{4 \alpha} \int_{x_{N}}^{x_{e n d}} d x\left(\frac{d \phi}{d x}\right)^{2}\left(b^{1 / 3} \cosh (2 x / 3)-1\right) \tanh x
$$

where $x_{\text {end }}$ denotes the value of $x$ when inflation ends. The amplitude of scalar perturbation is given by

$\delta_{H}^{2}=\left(\frac{1}{600 \pi^{2}} \frac{\kappa_{4}^{6} V^{8}}{\lambda^{3} V_{0} V_{, \phi}^{2}}\right)\left(\frac{729}{8 b}\right)\left(\frac{\left(b^{1 / 3} \cosh (2 x / 3)-1\right)^{3}}{\sinh ^{6} x}\right)$

using the canonically normalized field $\phi \rightarrow \sqrt{V_{o}} \phi[4]$. The exact expression for the amplitude of density perturbations found by Hwang and Noh [7], $\delta_{H}=H^{2} / 2 \pi \dot{\phi} \sqrt{V}$ is not very different from (18) as the tachyonic inflation commences very near to the top of the potential. 


\section{A. Exact Solution of Slow Roll Equations}

We now study evolution of the universe with tachyon field in an exponential potential which is of the form

$$
V=V_{o} e^{-\beta \kappa_{4} \phi},
$$

It would be interesting to obtain the exact solutions of slow roll equations in high and low energy limits. In high energy approximation $x>>1$, the Friedmann equation on the Gauss-Bonnet brane approximates to

$$
H^{2}=A \rho^{q}
$$

where $q=2 / 3$. We shall integrate the equations of motion in slow roll regime for arbitrary $q$. In case of the exponential potential, we have

$$
\dot{\phi}(t)=\frac{\beta}{3 \delta M_{p}} e^{\frac{\beta}{2 M_{p}} q \phi}
$$

where $\delta=\left(A V_{0}^{q}\right)^{1 / 2}$ Equations (20) and (21) are readily solved to yield

$$
\begin{gathered}
\phi(t)=-\frac{2 M_{p}}{\beta q} \ln \left[C-\frac{\beta^{2} q}{6 \delta M_{p}^{2}} t\right] \\
\frac{a(t)}{a_{i}}=e^{\delta\left(C-\frac{\beta^{2} q}{12 \delta M_{p}^{2}} t\right) t}
\end{gathered}
$$

where $\mathrm{C}$ is a constant determined by the initial value of $\phi$. The scale factor given by equation (23) passes through a point of inflection marking the end of inflation leading to

$$
\dot{\phi}_{\text {end }}=\sqrt{\frac{2}{3 q}}, \quad V_{\text {end }}=V_{0}\left(\frac{\beta q^{1 / 2}}{\sqrt{6} \delta M_{p}}\right)^{2 / q}
$$

One can arrive at (24) by demanding $\dot{H}+H^{2}>0$ without making use of slow roll equations. Since $q=2 / 3$ in the case under consideration, we find that $\dot{\phi}_{\text {end }}=1$. One should, however keep in mind, that Gauss-Bonnet Friedmann equation in the low energy limit $(x<<1)$ reduces to standard RS form and in case $\rho>>\lambda$ reduces to (20) with $q=2$ and $\delta=\left(1 / 6 \lambda M_{p}^{2}\right)^{1 / 2}$ leading to the following [4]

$$
\dot{\phi}_{\text {end }}=\sqrt{\frac{1}{3}}, \quad V_{\text {end }}=\beta \sqrt{2 \lambda}
$$

\section{B. Number of e-foldings and Density Perturbations}

We now compute the number of e-folds and density perturbation for the tachyonic system. For an exponential potential, the value of the field, $x_{N}$, corresponding to $N$ e-folds before the end of inflation is given by

$$
\begin{aligned}
N=-\frac{3}{4 \alpha} \int_{x_{N}}^{x_{\text {end }}} d x \frac{1}{\left(\beta \kappa_{4}\right)^{2}} \operatorname{coth}^{2} x\left(b^{1 / 3} \cosh (2 x / 3)-1\right) \tanh x . \alpha^{3} \lambda & =\frac{27 \times 10^{16}}{256 \pi^{2}} \kappa_{4}^{2}\left(\frac{2 N+1}{3(\ln 3-1)+2 f\left(x_{N}\right)}\right)^{2} \\
& \times\left(\cosh \left(2 x_{N} / 3\right)-1\right)^{6}
\end{aligned}
$$

The integral in (26) may be evaluated analytically, which becomes

$$
N=-\frac{3}{4 \alpha\left(\beta \kappa_{4}\right)^{2}}[f(x)]_{x_{N}}^{x_{e n d}}
$$

where we have

$$
\begin{aligned}
f(x) & =\frac{3}{2} b^{1 / 3} \cosh (2 x / 3)- \\
& -\frac{1}{2}\left(2+b^{1 / 3}\right) \ln [1+2 \cosh (2 x / 3)]+ \\
& +(b-1) \ln (\sinh (x / 3))
\end{aligned}
$$

It is possible to simplify the above expression if $\alpha \Lambda<<$ 1 which leads to $b \sim 1$. The end of the inflationary epoch is determined by noting that $x>>1$, the slow roll parameter $\epsilon>>1$. Thus one determines $x_{\text {end }}$ and $V_{\text {end }}$ from the condition that $x$ is sufficiently small (i.e., $x<<$ 1). Using equations (13) and (25), it is now possible to determine $x_{\text {end }}$

$$
x_{\text {end }}=\left(6 \alpha \beta^{2} \kappa_{4}^{2}\right)^{1 / 2} .
$$

Using equation (28) gives

$$
f\left(x_{e n d}\right)=\frac{3}{2}-\frac{3 \ln (3)}{3}+\frac{x_{e n d}^{2}}{9}
$$

Now equating (29) and (27) we determine $\alpha$

$$
\alpha=\frac{3}{4(2 N+1) \beta^{2} \kappa_{4}^{2}}\left[3(\ln 3-1)+2 f\left(x_{N}\right)\right]
$$

Using (18) and COBE normalization we obtain

$$
\alpha^{5} \lambda=\frac{243 \times 10^{16}}{4096 \kappa_{4}^{2} \pi^{2}} \frac{1}{\beta^{4}}\left[\cosh \left(2 x_{N} / 3\right)-1\right]^{3}
$$

One may now extract the values of the brane tension $\lambda$ and Gauss-Bonnet coupling, $\alpha$ which are consistent with the COBE normalization.

The slow roll parameters can now be cast in the form

$$
\epsilon=\left[\frac{\ln 3-1+\frac{2}{3} f\left(x_{N}\right)}{2 N+1}\right] \frac{\sinh \left(2 x_{N} / 3\right) \tanh \left(x_{N}\right)}{\left(\cosh \left(2 x_{N} / 3\right)-1\right)^{2}}
$$

while $\eta=0$ in case of exponential potential. To get scalar field confined on the brane we have to impose the constraint $\rho<\kappa_{5}^{-8 / 3}$ which leads to a lower limit on the allowed values of $\alpha^{3} \lambda$ for a given $x_{N}[1]$

$$
\alpha^{3} \lambda>48 \kappa_{4}^{2} \sinh ^{6} x_{N}
$$

Using equations (31) and (32) we determine 
The inequality (34) is satisfied for values of $x_{N}$ such that $x_{N} \leq 6.5$. Making use of (33), we find that the spectral index $n_{s} \simeq 0.97$ for an allowed value of $x_{N}$. Unfortunately, the numerical values of $\lambda$ and $V_{\text {end }}$ turn out to be larger than the Planck's scale if $\beta / M_{p}^{2} \sim 1$, i.e., if one restricts to string theory tachyons. This , of course, puts the whole procedure under doubt as the classical treatment of gravity becomes invalid in this case. Actually, the problem is present even at the RS level itself which, it seems, can not be corrected by Gauss-Bonnet correction. Indeed, the RS level expression for the asymmetry is given by,

$$
\delta_{H}^{2}=\frac{8 k_{4}^{4} \beta^{4} \times(2 N+1)^{5 / 2}}{600 \pi^{2} V_{\text {end }}} \simeq 10^{-10}
$$

where $V_{\text {end }}=V /(2 N+1)^{1 / 2}[4]$ in case of the exponential potential. It follows from equation (36) that $V_{\text {end }}>M_{p}^{4}$ if $\beta / M_{p}^{2} \simeq 1$. This is related to the fact that not enough inflation can be drawn in case of tachyonic field if one restricts to string theory tachyons. The situation could be remedied either by tuning the parameter $\beta$ or by introducing the large number of D-branes parallel to each other and separated by a distance much larger than $l_{s}$ (this is the same mechanism of assisted inflation as introduced by Liddle et. al $[8]$ ). The first option is out of place as $\beta$ is not a free parameter in tachyon potential $(\beta$ is fixed by sring scale $l_{s}$ ). As for the large number of D-brane assisted inflation is concerned, one could draw enough inflation in this procedure and push the spectral index $n_{s}$ close to one in the standard FRW cosmology itself. The sole purpose of invoking the brane worlds with Gauss-Bonnet term in the bulk is defeated. It is remarkable that the exponential potential, in case of normal scalar field, on the brane with Gauss-Bonnet Einstein equations in bulk allows to push $n_{s}$ very close to one independently of the slope of potential. It was, therefore, natural to investigate the tachyonic system along the same line as there is no free parameter in this case to tune. But due to the peculiar nature of tachyon field dynamics, the proposal of Lidsey and Nunes does not seem to work in a natural way.

\section{Acknowledgments}

We are thankful to J. E. Lidsey, N. J. Nunes and N. Dadhich for their critical comments. We also thank James Gregory, S. Odintsov and S. Panda for helpful comments.
[1] J. E. Lidsey and N. J. Nunes, Phys. Rev. D 67, 103510 (2003).

[2] A. Sen, hep-th/0203211 hep-th/0203265 hep-th/0204143

[3] Gibbons, G.W, arXiv:hep-th/0204008 A. Mazumdar, S. Panda, A. Perez-Lorenzana, hep-ph/0107058 Mukohyama, S hep-th/0204084 Frolov, A, L. Kofman and A. Starobinsky, Phys.Lett. B545, 8 (2002); Choudhury, D, D. Ghoshal, D. P. Jatkar and S. Panda, hep-th/0204204 G Shiu and I. Wasserman, Phys.Lett. B541 (2002) 6; Padmanabhan, T., Phys. Rev. D66, 021301(2002); T. Padmanabhan, T. Roy Choudhury, hep-th/0205055 Kofman, L and A. Linde, arXiv: hep-th/0205121 Sami, M., hep-th/0205146 Piao, Y.S, R. G. Cai, X. m. Zhang and Y. Z. Zhang, hep-ph/0207143 Cline, J.M, H. Firouzjahi and P. Martineau, hep-th/0207156 Zong-Kuan Guo, Yun-Song Piao, Rong-Gen Cai, Yuan-Zhong Zhang, hep-ph/0304236 Yun-Song Piao, Rong-Gen Cai, Xinmin Zhang, Yuan-Zhong Zhang, hep-ph/0207143 G. Felder, Lev Kofman and A. Starobinsky, JHEP 0209 (2002) 026. Wang, B, E. Abdalla and R. K. Su, hep-th/0208023 Jian-gang Hao, Xin-zhou Li, hep-th/0209041 M. C. Bento, O. Bertolami and A.A. Sen, hep-th/020812; M.C. Bento, O. Bertolami., A.A. Sen, Phys.Rev.D67:023504,2003; gr-qc/0204046 M.C. Bento, O. Bertolami., Phys.Rev.D65:063513,2002; Jian-gang Hao, Xin-zhou Li, Phys.Rev. D66 (2002) 087301; Chanju Kim , Hang Bae Kim and Yoonbai Kim, hep-th/0210101 J.S.Bagla, H.K.Jassal, T.Padmanabhan, astro-ph/0212198 M. Sami, P. Chingangbam and T. Qureshi, hep-th/0301140 Xin-zhou
Li, Dao-jun Liu, Jian-gang Hao, hep-th/0207146 M. Majumdar, Anne-Christine Davis, hep-th/0304226 D. Choudhury, D. Ghoshal, D. P. Jatkar, S. Panda, hep-th/0305104 D.A.Steer, F.Vernizzi, hep-th/0310139 Zong-Kuan Guo, Hong-Sheng Zhang, Yuan-Zhong Zhang, hep-ph/0309163 ; P.K.Suresh, gr-qc/0309043 M. R. Garousi hep-th/0307197 Maria C. Bento, Nuno M. C. Santos, A. A. Sen, astro-ph/0307292 Yun-Song Piao, Yuan-Zhong Zhang,. hep-th/0307074 ; D. Bazeia, F.A. Brito, J.R. Nascimento, hep-th/0306284 Shin'ichi Nojiri, Sergei D. Odintsov, hep-th/0306212 Jian-gang Hao, Xin-zhou Li,. hep-th/0305207 L. Raul W. Abramo, Fabio Finelli, astro-ph/0307208 V. Gorini, A. Yu. Kamenshchik and U. Moschella, V. Pasquier, hep-th/0311111 Marie-Bernadette Causse, astro-ph/0312206 Dao-jun Liu, Xin-zhou Li, astro-ph/0402063 J.M. Aguirregabiria, Ruth Lazkoz, hep-th/0402190 J.M. Aguirregabiria, Ruth Lazkoz, gr-qc/0402060 ; Gianluca Calcagni, hep-ph/0402126 Kenji Hotta, hep-th/0403078 ; Xin-He Meng, Peng Wang, hep-ph/0312113 M. R. Garousi, M. Sami and Shinji Tsujikawa, hep-th/0402075 Luis P. Chimento, astro-ph/0311613.

[4] Fairbairn M and M. H. Tytgat, hep-th/0204070 Sami, M, P. Chingangbam and T. Qureshi, hep-th/0205179

[5] B. Zwiebach, in Anomalies, Geometry and Topology - proceedings of the symposium, Argonne, Illinois, 1985, edited by W. A. Bar; D. Lorentz-Petzold, Prog. Theor. Phys. 78, 969 (1987);Mod. Phys. Lett. A 3, 827 (1988); A. B. Henriques, Nucl. Phys. 277 B, 621 (1986); Q. Shafi and C. Wetterich, Phys. Lett. 129 B, 387 (1983); ibid 152 B, 51 (1984); H. Ishihara, Phys. Lett. 179 B, 217 (1986); B. C. 
Paul and S. Mukherjee, Phys. Rev. D 42, 2595 (1990); I. P. Neupane, hep-th/0108194 I. P. Neupane, hep-th/0106100

[6] Shin'ichi Nojiri, Sergei D. Odintsov, hep-th/0006232 B. Abdesselam and N. Mohammedi, Phys. Rev. D 65, 084018 (2002); J. E. Lidsey, Shin'ichi Nojiri, S. D. Odintsov, hep-th/0202198 C. Charmouses and J. Dufaux, hep-th/0202107 S.C. Devis, hep-th/0208205 E. Gravanis and S. Willison, hep-th/0209076 S. Nojiri, S.Odintsov and S. Ogushi, Phys. Rev. D 65 (2002) 023521; J. P. Gregory, A. Padilla, hep-th/0304250 H.
M. Lee, hep-th/0010193 (2000); J. E. Kim and H. M. Lee, gr-qc/0306116 (2003); N. Deruelle and C. Germani, gr-qc/0306116 (2003); Nathalie Deruelle and John Madore, gr-qc/0305004 Kei-ichi Maeda, Takashi Torii, hep-th/0309152

[7] J. Hwang and H. Noh, hep-th/0206100

[8] Andrew R. Liddle, A. Mazumdar, F. E. Schunck, astro-ph/9804177 